\title{
PLEURAL TENTING DURING UPPER LOBECTOMY DECREASES CHEST TUBE TIME AND TOTAL HOSPITALIZATION DAYS
}

Lary A. Robinson, MD

Dianne Preksto, PA-C

\begin{abstract}
Objective: A prolonged air leak after an upper lobectomy is a major determinant of morbidity and hospital stay. Creation of a pleural tent after upper lobectomy was used to investigate whether obliterating the usual postoperative intrapleural apical space with the parietal pleura would help shorten chest tube time. Methods: From August, 1994, through January, 1997, 48 consecutive patients undergoing an isolated upper lobectomy for a neoplasm were reviewed. Twenty-eight patients had creation of a pleural tent and 20 patients did not. Demographic and clinical profiles of both groups were not significantly different. Chest tubes were removed when there was no air leak for $\mathbf{4 8}$ hours and chest tube drainage was less than $\mathbf{7 5}$ ml per 8 hours. Results: The tented patients had significantly shorter mean air leak (tented $1.6 \pm 0.3$ days vs nontented $3.9 \pm 1.2$ days, $p=0.04$ ), mean chest tube total drainage (tented $1619.5 \pm 95.5 \mathrm{ml}$ vs nontented $2476.3 \pm$ $346.4 \mathrm{ml}, p=0.009$ ), mean chest tube duration (tented $4.0 \pm 0.2$ days vs nontented $6.6 \pm 1.0$ days, $p=0.004$ ), mean total hospitalization time (tented $6.4 \pm 0.4$ days vs nontented $8.6 \pm 1.0$ days, $p=0.02$ ). No operative deaths occurred. Morbidity was not significantly different between groups. Conclusions: (1) Creation of a pleural tent at the time of upper lobectomy appears to significantly reduce chest tube time and shorten hospitalization. (2) No morbidity or mortality was associated with this simple, quick procedure. (3) Surgeons should consider creation of a pleural tent at the time of upper lobectomy. (J Thorac Cardiovasc Surg 1998;115:319-27)
\end{abstract}

"If the hill will not come to Mahomet [Mohammed], Mahomet will go to the hill."*

\section{Francis Bacon (1561-1626) Essays, Of Boldness ${ }^{1}$}

The he development of a pleural space after a major lung resection has been the subject of concern and attempts at prevention ever since the beginning

From the Division of Cardiovascular and Thoracic Surgery, H. Lee Moffitt Cancer Center and Research Institute, University of South Florida College of Medicine, Tampa, Fla.

Read at the Seventy-seventh Annual Meeting of The American Association for Thoracic Surgery, Washington, D.C., May 4-7, 1997.

Received for publication May 7, 1997; revisions requested July 15, 1997; revisions received Oct. 7, 1997; accepted for publication Oct. 7, 1997.

Address for reprints: Lary A. Robinson, MD, H. Lee Moffitt Cancer Center and Research Institute, 12902 Magnolia Dr., Tampa, FL 33612-9497.

* Often misquoted as "If the mountain will not come to Mohammed...."

Copyright (C) 1998 by Mosby, Inc.

$0022-5223 / 98 \$ 5.00+0 \quad \mathbf{1 2 / 6 / 8 6 7 2 4}$ of pulmonary surgery early in this century. ${ }^{2,3}$ Initially it was thought that a residual pleural space was an ominous sign with the likely result to be infection and potential mortality. Only later was it realized that these spaces were not so dangerous. Although they were frequent, they only potentially led to morbidity with prolonged air leaks and an occasional empyema. By standard chest radiography, $20 \%$ to $40 \%$ of all patients having major lung resection will initially have a pleural space. But with more sophisticated imaging, it is apparent that most patients will have a space and virtually all undergoing an upper lobectomy will have an anterior and apical space. ${ }^{2}$

Because almost all pulmonary resections require cutting across lung parenchyma to develop fissures or planes, which is commonly performed with an automatic lung stapling device, there is potential for an air leak, which usually seals soon. Air leaks seal most rapidly when the defect or hole in the visceral pleura is brought directly in opposition with the parietal pleura by suction such that the lung surface is "patched" by the parietal pleura. ${ }^{4}$ In the patient 
having a pulmonary resection, and especially an upper lobectomy with a large air space (commonly with two fissure staple lines in this space) under chest tube suction, the two pleural surfaces will not approximate. This often leads to a frustratingly prolonged air leak with its increased morbidity and hospitalization time, resulting in a mean 5.6 extra hospital days reported in one study. ${ }^{5}$

Numerous measures have been advocated to prevent residual pleural spaces and the common accompanying prolonged air leak, including thoracoplasty, pneumoperitoneum, pleural decortication, phrenic nerve crush, muscle flap transposition, high intrapleural suction, pleural partition, phrenoplasty (Lyman Brewer maneuver), and "meticulous surgical technique."2,3,5 One other simple technique described initially in $1956^{6}$ and later further advocated $^{7,8}$ is creation of the apical pleural tent. This procedure has significant theoretic appeal because when the visceral pleura will not rise up to the parietal pleura, we can drop down the parietal pleura to the lung to reapproximate the two pleural surfaces and seal the air leak, thereby obliterating the residual intrapleural air space. Despite the enthusiasm at the early description of this procedure, little has been written about the pleural tent since 1963 except for an occasional brief mention in review articles. ${ }^{2,3,5}$ Moreover, no comparative studies have been published documenting the effectiveness of this technique as an adjunct to modern pulmonary resections.

In view of the common problem of prolonged air leaks in patients undergoing upper lobectomy for malignancy, we elected to add the routine creation of an apical pleural tent to the end of the surgery and compare the results with nontented upper lobectomies. It was our hypothesis that reapproximating the pleural surfaces would seal air leaks quicker, allow earlier chest tube removal, and result in shorter hospitalizations.

\section{Methods}

Patient population. The records of 63 consecutive patients undergoing an upper lobectomy for a neoplasm in a single teaching hospital setting by the same surgeon (L.R.) from August 1994 through January 1997 were retrospectively reviewed. Fifteen patients were excluded if they had conditions that might promote or decrease an air leak or lead to prolongation of chest tube duration: concomitant chest wall resection (eight patients), requirement for postoperative mechanical ventilation (one patient), alcohol withdrawal syndrome (two patients), or extensive pleural adhesions (four patients). Patients undergoing surgery during the first year of the study period underwent a standard upper lobectomy only (nontented patient group, $n=20$ ). Subsequently, all patients underwent the same surgical procedure but additionally had creation of an apical pleural tent ${ }^{6,7}$ just before wound closure (tented patient group, $n=28$ ). The clinical profiles and perioperative course of the patients in the nontented group was compared with the later group of patients treated with a tent to evaluate the effects of this adjunctive measure on air leak and chest tube duration, morbidity, and the length of hospitalization.

Operative technique. All lobectomies are performed using a posterolateral muscle-sparing thoracotomy (sparing the latissimus dorsi and serratus anterior muscles) entering the chest through the fifth intercostal space. All patients receive preoperative prophylactic heparin, 5000 units subcutaneously, and this is continued every 12 hours during the postoperative period. In addition, patients receive one preoperative and two postoperative intravenous doses of prophylactic cefuroxime except in the penicillin-allergic patients who receive vancomycin. No further antibiotics are given unless there is a specific treatable condition such as a respiratory infection. Full-leg length intermittent pneumatic compression stockings are used during the operation and for 3 days after the operation on all patients. A mediastinal lymphadenectomy is performed on all cases. Mechanical stapling devices are used routinely to develop incomplete fissures and to close the bronchus. Intraoperatively, bronchial stumps are tested for an air leak with $30 \mathrm{~cm} \mathrm{H}_{2} \mathrm{O}$ airway pressure while the stump is submersed under saline solution. After ensuring an airtight closure, a pleural flap mobilized inferiorly and separately from the pleural tent is sutured over the bronchial stump in all patients. The inferior pulmonary ligament is fully transected up to the inferior pulmonary vein to ensure maximal mobilization of the remaining lung after resection to minimize a residual air space. Postoperative pain control is provided by continuous epidural catheter analgesia. Postoperatively, chest tubes are removed when there was no air leak for 48 hours and the total chest tube drainage is less than $75 \mathrm{ml}$ for an 8-hour period.

In this study, pleural tents are created in patients generally using the technique described in 1956 by Miscall and associates ${ }^{6}$ and more recently by J. I. Miller. ${ }^{3}$ The parietal pleura is mobilized starting along the upper border of the thoracotomy incision posteriorly where the pleura is thicker. The parietal pleura is best held by the surgeon during mobilization with Singley (ring) forceps to avoid tearing this delicate structure. Particular care should be exercised dissecting away the pleura anteriorly because it usually is much thinner and more fragile. The pleura is dissected down from the endothoracic fascia of the thoracic wall and apex but not off the phrenic nerve or mediastinum. The extrapleural dissection is best performed under direct vision using a Kitner dissector to minimize trauma to the pleura. A generous area of parietal pleura is carefully dissected down such that when released it will fall completely covering the mediastinum. If the dissection is done carefully, there is rarely any significant bleeding from the chest wall and what little there is may be easily controlled with the electrocautery. 
Inadvertent small tears in the tent may be closed in part with appropriately placed small hemoclips. After mobilization is completed, the midportion of the lower edge of the free pleural margin is then attached in one place to the superior margin of the intercostal muscle at the chest wound with a hemoclip. Thus the pleural tent actually has the appearance of a tent as it is suspended to cover the raw lung parenchyma after the resection when the remaining lung is reinflated. Alternatively, the lowest free edge of the parietal pleura may be caught by one of the paracostal sutures used to reapproximate the ribs to suspend the pleural tent such that the reinflated lung travels superiorly up under the tent. Two $28 \mathrm{~F}$ chest tubes are inserted routinely through separate stab wounds inferior to the thoracotomy wound and are directed anteriorly and posteriorly in the chest under the tent along with the reexpanded lung. After thoracotomy closure, the chest tubes are placed on continuous underwater seal suction at 10 to $20 \mathrm{~cm} \mathrm{H}_{2} \mathrm{O}$. In so doing, the pleural tent is sucked down over the staple lines on the remaining lung. A JacksonPratt drain is inserted into the subcutaneous space under the skin flaps created during the muscle-sparing thoracotomy.

An operative mortality is considered to be any occurring within 30 days after the operation. For the purposes of presenting morbidities, purulent bronchitis is defined as relatively abrupt onset of a cough productive of purulent sputum usually associated with a low-grade fever but no infiltrate is seen on chest radiographs. To be considered a pneumonia, an infiltrate must be seen on a chest radiograph, and it is usually associated with a leukocytosis and increased inspired oxygen requirements.

Statistical evaluation. All numerical patient data are expressed as the mean \pm the standard error of the mean. Comparisons between the two groups are made using analysis of variance (unpaired Student's $t$ test) with a computer-driven statistical program ${ }^{9}$ on a Macintosh II computer (Apple Computer, Cupertino, Calif.).

\section{Results}

The characteristics of the patients, including preoperative pulmonary function studies in both groups, are displayed in Table I and show no significant differences. One patient in each group was receiving systemic corticosteroid treatment at the time of thoracotomy.

Operative data. The total mean combined surgical and anesthesia times (total time in the operating room) in the two groups were $219.2 \pm 11.2$ minutes (range 160 to 350 minutes) in the tented group, which was significantly less than $274.0 \pm 15.2$ minutes (range 175 to 430 minutes) in the nontented group $(p=0.005)$. During the earlier part of the study period (when the nontented cases where being accumulated), the epidural catheter was placed by the anesthesiologist just after the patient entered the operating room, thereby increasing the overall time. Later in the study period, procedures were
Table I. Preoperative patient profiles

\begin{tabular}{lccc}
\hline \multicolumn{1}{c}{ Parameter } & $\begin{array}{c}\text { Tented } \\
(n=28)\end{array}$ & $\begin{array}{c}\text { Nontented } \\
(n=20)\end{array}$ & $p$ Value \\
\hline Age (yr) & $65.8 \pm 1.8$ & $62.8 \pm 3.0$ & 0.38 \\
Male/female & $18 / 10$ & $13 / 7$ & - \\
FEV $_{1}$ (liters) & $2.18 \pm 0.12$ & $2.51 \pm 0.19$ & 0.12 \\
FEV $_{1}$ (\% predicted) & $76.3 \pm 4.1$ & $72.9 \pm 5.3$ & 0.60 \\
DLCO $_{\text {corr }}$ & $74.0 \pm 4.2$ & $70.9 \pm 4.6$ & 0.62 \\
$\quad$ \% predicted) & & & \\
$\mathrm{FEV}_{1} / \mathrm{FVC}(\%)$ & $67.0 \pm 2.4$ & $66.1 \pm 3.3$ & 0.83 \\
$\mathrm{FEV}_{25-75}$ (liters) & $1.53 \pm 0.2$ & $1.79 \pm 0.3$ & 0.42 \\
$\mathrm{FEV}_{25-75}$ & $51.0 \pm 6.0$ & $54.9 \pm 7.1$ & 0.67 \\
$\quad(\%$ predicted) & & & \\
$\mathrm{PO}_{2}$ (mm Hg) & $83.2 \pm 1.8$ & $80.8 \pm 2.3$ & 0.42
\end{tabular}

$F E V_{1}$, Forced expiratory volume in one second; $D L C O_{c o r r}$, diffusing capacity of carbon monoxide corrected for alveolar ventilation; $F E V_{1} / F V C$, forced expiratory volume at one second divided by the forced vital capacity, expressed as a percentage; $F E V_{25-75}$, forced expiratory volume between $25 \%$ to $75 \%$ of vital capacity.

changed so that the epidural catheter was inserted while the patient was still in the preoperative area, resulting in less total time in the operating room. This change probably accounts for most of the difference in the combined surgical and anesthesia times of the two groups listed above. During the learning period in the first few cases, approximately 10 minutes was required to create a pleural tent. Currently, the average time is 4 minutes and has no impact on overall operative times.

Surgical procedures performed in the nontented group were 10/20 (50.0\%) right and 10/20 (50.0\%) left upper lobectomies. In the tented group there were 20/28 (71.4\%) right upper lobectomies and $8 / 28$ (28.6\%) left upper lobectomies. All patients had bronchoscopy performed before the thoracotomy, and five patients in the nontented and four patients in the tented groups also had mediastinoscopy performed just before the thoracotomy. All patients had a mediastinal lymphadenectomy performed at the time of lobectomy.

Pathology. The final pathologic staging of the lung cancer patients was similar for both groups: stage I 20/28 (71.4\%) tented, 12/20 (60.0\%) nontented; stage II 1/28 (3.6\%) tented, $2 / 20(10.0 \%)$ nontented; stage IIIA 5/28 (17.9\%) tented, 4/20 $(20 \%)$ nontented; and stage IV 1/20 (5.0\%) in the nontented group only. The cell types of the resected tumors in the tented group were adenocarcinoma $(\mathrm{n}=18)$, squamous cell carcinoma $(n=6)$, large cell undifferentiated carcinoma $(n=1)$, neuroendocrine carcinoma $(n=1)$, and metastatic carcinoma (colon adenocarcinoma and laryngeal squamous cell carcinoma; $n=2$ ). The cell types for the nontented 


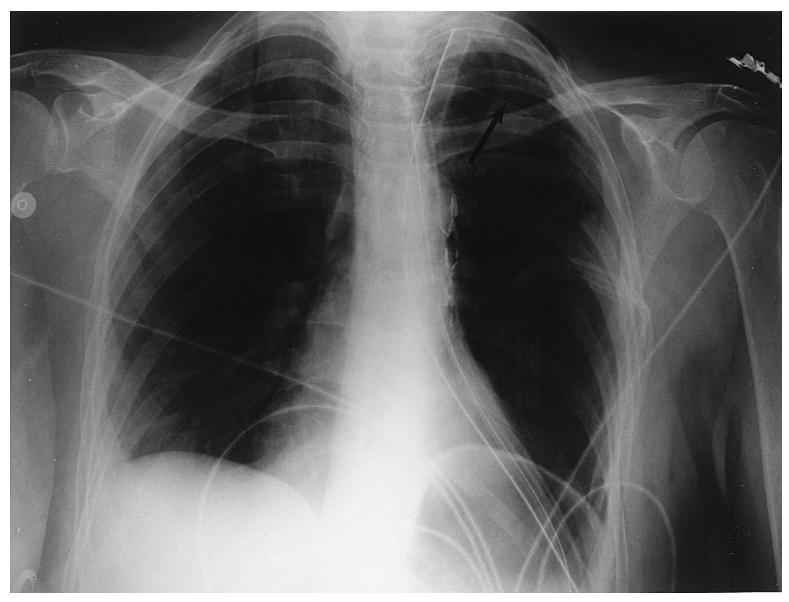

Fig. 1. Anteroposterior portable chest radiograph taken immediately after the operation in an patient with an upper lobectomy in the group having a pleural tent. The apical extrapleural air space is readily apparent with the arrow demonstrating the apex of the remaining lung. The pleural tent resting on top of the lung and chest tubes is not radiographically visible. The chest tubes are intrapleural. The Jackson-Pratt drain is located subcutaneously under the skin flaps raised during the muscle-sparing thoracotomy.

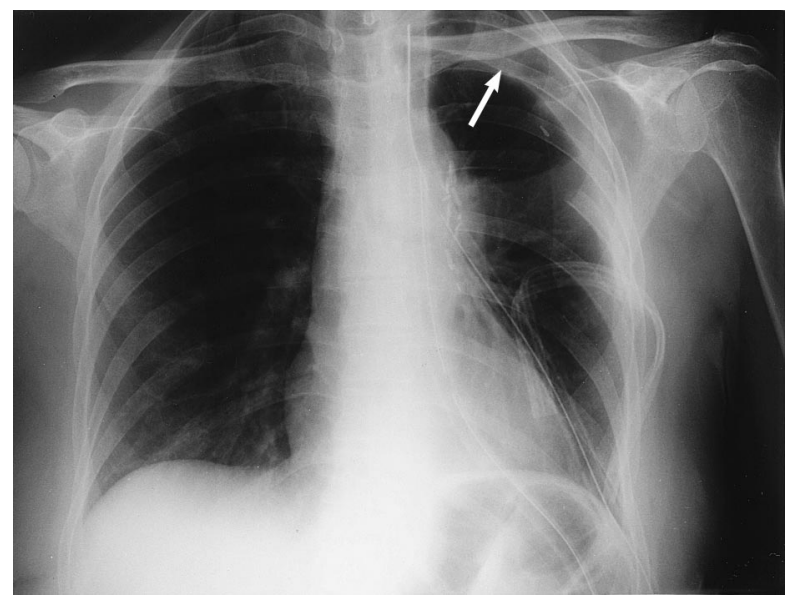

Fig. 2. Anteroposterior portable chest radiograph of the same patient 4 days later just before chest tube removal, demonstrating the same apical extrapleural air space is still present and more readily visible (marked by the arrow) but the air leak had ceased 2 days earlier.

group were adenocarcinoma $(n=9)$, squamous cell carcinoma $(n=7)$, large cell undifferentiated carcinoma $(n=1)$, carcinoid tumor $(n=2)$, and metastatic renal cell carcinoma $(n=1)$.

Complications. The perioperative morbidity and mortality are shown in Table II. No significant
Table II. Morbidity and mortality

\begin{tabular}{lll}
\hline \multicolumn{1}{c}{ Complication } & $\begin{array}{c}\text { Tented } \\
(n=28)\end{array}$ & $\begin{array}{l}\text { Nontented } \\
(n=20)\end{array}$ \\
\hline Bleeding requiring reoperation & 0 & 0 \\
Need for chest tube reinsertion & 0 & 0 \\
Pleural infection or empyema & 0 & 0 \\
Wound infection & 0 & 0 \\
Atelectasis requiring bronchoscopy & 0 & 0 \\
Purulent bronchitis & $4(14.3 \%)$ & $1(5.0 \%)$ \\
Pneumonia & 0 & 0 \\
Atrial arrhythmia & $1(3 \%)$ & $2(10 \%)$ \\
Transfusion & 0 & 0 \\
Stroke & 0 & 0 \\
Myocardial event & 0 & 0 \\
Venous thrombosis or embolus & 0 & 0 \\
Late complications after discharge & 0 & 0 \\
Mortality & 0 & 0 \\
\hline
\end{tabular}

differences were found between the two groups. No morbidity was associated with creation of the pleural tent.

Radiographic findings. Almost all patients undergoing an upper lobectomy in both groups had a radiographically demonstrable apical air space postoperatively. Fig. 1 displays the appearance of the initial postoperative portable chest radiograph in a typical patient in the tented group, with the apical (extrapleural) air space easily visible, but the pleura tent draped over the lung is usually not seen. Fig. 2 shows the same patient 4 days later just before chest tube removal (after 48 hours of no air leak), showing that the extrapleural apical air space is still readily visible but the air leak ceased on the second postoperative day. Fig. 3 shows the same patient 1 month later illustrating the usual resolution of the apical air space to only residual pleural thickening (so-called pleural cap), similar to that described by Goodman and associates. ${ }^{10}$

In some patients, the apical extrapleural space initially remained open with air even after the air leak ceased. After chest tube removal, the space then progressively filled with fluid, which was seen as an apical air-fluid level on chest radiograph. After 4 to 6 weeks, the space opacified, and over subsequent months it contracted to the point where only slight apical pleural thickening remained very similar to that seen in patients undergoing a nontented upper lobectomy. In other patients the extrapleural space opacified rapidly before chest tube removal and then it followed the same radiographic pattern of resolution.

Chest tube management data. Table III lists the comparison of the air leak and chest tube manage- 
Table III. Postoperative management data

\begin{tabular}{lccc}
\hline \multicolumn{1}{c}{ Parameter } & $\begin{array}{c}\text { Tented } \\
(n=28)\end{array}$ & $\begin{array}{c}\text { Nontented } \\
(n=20)\end{array}$ & $p$ Value \\
\hline $\begin{array}{l}\text { Air leak after surgery } \\
\text { (days) }\end{array}$ & $1.61 \pm 0.3$ & $3.85 \pm 1.2$ & 0.02 \\
$\begin{array}{c}\text { Total chest tube } \\
\text { drainage (ml) }\end{array}$ & $1619.5 \pm 95.5$ & $2476.3 \pm 346.4$ & 0.009 \\
$\begin{array}{c}\text { Total days chest tubes } \\
\text { in place }\end{array}$ & $4.0 \pm 0.2$ & $6.6 \pm 1.0$ & 0.004 \\
\begin{tabular}{l} 
Total days in hospital \\
\hline
\end{tabular} & $6.4 \pm 0.4$ & $8.6 \pm 1.0$ & 0.02 \\
\hline
\end{tabular}

ment data. The tented patients had significantly fewer days of air leak, chest tube drainage, chest tube duration, and more than 2 days less hospitalization compared with the nontented patients.

Hospitalization costs. Patients having a pleural tent created at the time of upper lobectomy had a mean 2 hospital days less $(6.4 \pm 0.4$ days tented vs $8.6 \pm 1.0$ days nontented, $p=0.02)$ than patients not having a pleural tent (Table III). The tented group's mean hospital stay of 6.4 days compares quite favorably with the national mean hospital stay for a lobectomy of 8.8 days as listed in St. Anthony's DRG Optimizer. ${ }^{11}$ That comparison is even more dramatic in Florida where the mean hospital stay for a lobectomy is 12.2 days (DRG-75 lobectomy, Florida Agency of Health Care Administration, Tallahassee, Fla.).

The total mean hospitalization charges (excluding professional fees for the surgeon and anesthesiologist) for the tented group was $\$ 21,432 \pm \$ 1334$ (median $\$ 21,263$, range $\$ 16,118$ to $\$ 36,664$ ) compared with $\$ 23,397 \pm \$ 789$ (median $\$ 22,004$, range $\$ 17,055$ to $\$ 40,375$ ) in the nontented group. However, because of a few outliers in each group and relatively small numbers of patients, this charge difference did not achieve statistical significance $(p=0.18)$. In comparison, these charges are also quite low compared with $\$ 42,080$, the mean Florida hospital charge for a lobectomy (DRG-75 lobectomy, Florida Agency of Health Care Administration, Tallahassee, Fla.).

\section{Discussion}

A residual intrapleural air space after a partial pulmonary resection has been a concern throughout the history of thoracic surgery because it is believed to invite complications from infection, bleeding, and prolonged air leak. Mechanisms for physiologic compensation seen initially after a partial lung resection that tend to obliterate the free pleural space

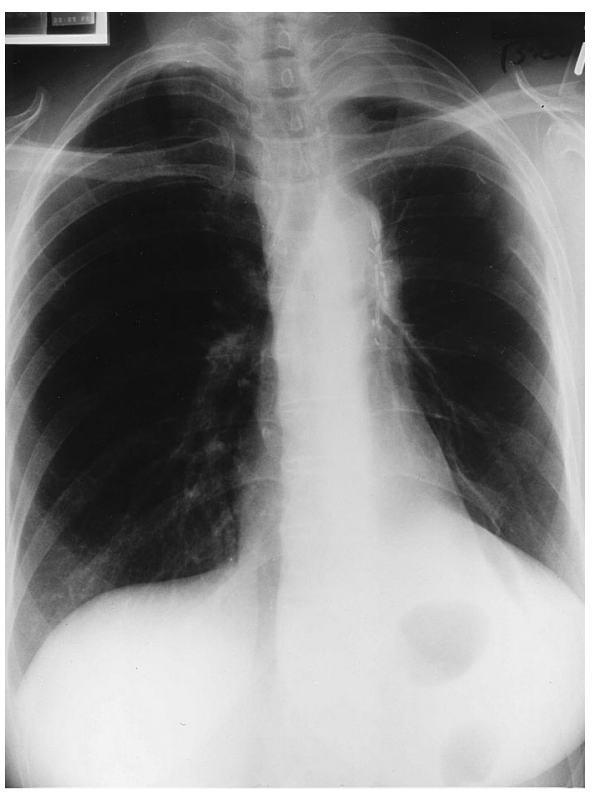

Fig. 3. Posteroanterior chest radiograph of the same patient as an outpatient 1 month later demonstrating the typical resolution of the apical extrapleural air space to only residual pleural thickening.

include: (1) hyperexpansion of the remaining lung and some remodeling of the lung shape to fit the space; (2) shift of the mediastinal structures to the operated side; (3) elevation of the ipsilateral diaphragm; and (4) narrowing of the ipsilateral intercostal spaces if the chest wall is not involved with restrictive disease. ${ }^{2}$ Most patients undergoing a lung resection have some degree of chronic obstructive lung disease and their lungs are already hyperexpanded, which negates the effectiveness of the first compensatory mechanism. Prior mediastinal radiation therapy or chest surgery such as coronary artery bypass grafting, which is exceedingly common in the lung cancer population, often results in a relatively immobile mediastinum, thereby diminishing the effect of the second space-decreasing mechanism listed above. Finally, the most common definitive operative procedure for lung cancer is an upper lobectomy because most primary lung cancers arise in the upper lobes. Resection of the upper lobe of the lung leaves behind a very irregular lung surface, which rarely conforms to the apex of the pleural cavity. Therefore, despite the compensatory mechanisms, the net result is the very high incidence of residual postresection apical pleural air spaces especially after upper lobectomy. With modern radiographic techniques, it is apparent that virtually all 
patients undergoing an upper lobectomy will have an anterior and apical residual air space to some extent. $^{2}$

In the early era of lung resections performed mostly for tuberculosis, "fear of the unclosed pleural space and the possibly adverse effects of [lung] overdistension" 1 led to the preference for preresectional or even concomitant thoracoplasty. ${ }^{2,7}$ Thoracoplasty was effective in preventing the residual pleural space but there was a great deal of morbidity from this procedure, including increased blood loss and operating time, prolonged hospitalization, and the resulting anatomic deformity that included significant scoliosis.

Surgeons looked to other less morbid approaches to prevent the postresectional space. The phrenic nerve was crushed during the operation to cause a temporary (ideally) diaphragmatic palsy to elevate the diaphragm. However, this maneuver interferes with respiratory function in many patients, especially those with chronic obstructive lung disease, and therefore the phrenic nerve crush fell out of favor. Intrapleural plombage with Lucite balls (also lipoid tissue, gauze packs, rubber sheeting and balloons, muscle flaps, paraffin, air, oil, or various plastics and metals) proved useful occasionally but generally the results of this technique were disappointing. ${ }^{6,7}$ Pneumoperitoneum was used to raise the diaphragm by increasing the intraabdominal pressure but probably it has little positive effect in reducing pleural space particularly with an upper lobectomy.

During the same time period in 1956, Brewer and associates $^{12}$ reported their experience partitioning the pleural cavity with fascia lata, whereas others described the use of Marlex mesh (Bard Implants, Billerica, Mass.), nylon mesh, tantalum, or steel for this purpose. ${ }^{13}$ None proved practical for the long term. Later, Brewer and Gazzaniga ${ }^{14}$ proposed another method to reduce the postresection pleural space, which they called phrenoplasty. This procedure involved dividing the diaphragmatic-pericardial attachments allowing the hemidiaphragm to rise, thereby further reducing the pleural dead space. This technique decreases the pleural space about $15 \%$ on the left but only $5 \%$ on the right. Because of the lack of proven benefit, phrenoplasty never gained widespread acceptance.

Perhaps the simplest adjunctive surgical procedure developed to reduce intrapleural postresection dead space is creation of the pleural tent, first described in 1956 by Miscal and associates. ${ }^{6}$ On the basis of the model of tailoring the thorax by thora- coplasty, Miscal began to mobilize the usually thickened, apical parietal pleura on lung resections for tuberculosis and then tented the pleura over the residual lung tissue by suturing the pleura to the lower edge of the wound. They began to use this technique beginning in 1948 and reported excellent results in their first 100 patients in their 1956 paper. Hansen in 1957 independently reported similar excellent results in his series of 22 patients using parietal pleurolysis (his name for the pleural tent). ${ }^{7}$ Bell in 1956 also used this technique successfully in 25 patients, but he also believed that the pleural tent "has assisted in reducing the incidence of transient air leakage from the raw surface of the lungs with limited pneumatic (self-sealing) function."15 Bell further emphasized that there was no pathologic or clinical evidence that adding thoracoplasty to decrease the residual pleural space made the lung resection safer for the patient.

Rainer and Newby ${ }^{8}$ in 1968 described a more complicated form of the pleural tent that they called extrapleural apicolysis, which had the same purpose but required mobilization of the endothoracic fascia along with the parietal pleura. This modification of the pleural tent was less acceptable because of the markedly increased bleeding that occurs with freeing up a large area of the endothoracic fascia. The simpler parietal pleural tent remained in the thoracic surgeon's armamentarium but it was only rarely used. Little was mentioned about this technique in the literature over the last 30 years save an occasional brief reference in a few review papers., 3,5 Just recently, with the advent of lung volume reduction surgery, a sizable apical air space is often created after linear resection of the apical portion of an emphysematous lung. Several investigators, including Cooper and associates, ${ }^{16}$ report that they commonly create a pleural tent at the end of the lung volume reduction procedure to allow visceral and parietal pleural apposition. However, they have not systematically examined the value of this procedure in their patient population.

On the basis of the early observations by Bell, ${ }^{15}$ Hansen, ${ }^{7}$ and Miscall and associates, ${ }^{6}$ regarding the ease of creating a pleural tent and particularly how it seemed to accelerate sealing leaks from the lung, we chose to use this method as an adjunct to upper lobectomy, which were the most likely patients to have a prolonged air leak and an apical space. Rice and Kirby ${ }^{5}$ in their review of prolonged postresection air leaks state a basic pulmonary surgeon's rule that "apposition of the raw operative surface of the lung to the parietal pleura assists in the closure of 
small bronchoalveolar pleural fistulas that have not or could not be surgically closed."

The healing process to seal air leaks from staple lines or raw surface requires parietal-to-visceral pleural contact. Because this apposition is usually not possible in the patient having an upper lobectomy with their postresection air space, then bringing down the parietal pleura to the lung surface by creating a pleural tent should serve the same purpose. This study substantiates this theory by demonstrating a significant reduction of the total number of days of postoperative air leak in a group of patients with upper lobectomy undergoing creation of a pleural tent compared with a comparable group of nontented patients. But more impressive is the $26 \%$ reduction in the mean hospital stay (Table III) in the tented patient group, which makes this procedure quite cost-effective. In a time of limited health care resources, a simple surgical technique such as the pleural tent that appears to decrease hospital stays and costs deserves careful consideration. Although for the purposes of uniformity in the study, we only compared patients with upper lobectomies; the pleural tent technique in our experience appears to provide the same positive lung sealing effect for less extensive resections of the upper lobe such as a segmentectomy or a large wedge resection.

The pleural tent procedure is simple, very quick to perform, and in this study was not associated with any morbidity. Theoretically, mobilization of the apical parietal pleura to create a tent could cause a hemorrhagic complication. However, if the surgeon carries out the procedure carefully under direct vision as previously described and remains in the extrapleural space leaving the endothoracic fascia intact, only tiny pleural vessels are disrupted that usually stop bleeding spontaneously or at most are easily controlled with point use of the electrocautery. Development of an empyema in the pleural tent space is theoretically possible but probably is no more likely to occur than in the intrapleural apical space remaining after an upper lobectomy without a pleural tent. Even in Miscal and associates' original series $^{6}$ of 99 patients undergoing upper lobectomy for infection (mostly tuberculosis) published 41 years ago, only two patients had a postoperative extrapleural infection, with one of these having a large bronchopleural fistula. Finally, the late apical thickening (so-called pleural cap) seen on the chest radiograph after a pleural tent procedure is no different than that seen after an upper lobectomy without a tent. Whether a later "redo" thoracotomy will be more difficult after a pleural tent remains to be seen because we have not reoperated on any of these patients. We strongly doubt that a prior pleural tent will make chest reentry any more difficult for a completion pneumonectomy, for example, than in a patient with a prior nontented upper lobectomy whose apical fluid-filled intrapleural space healed with the usual fibrosis.

The current series has several limitations, including the fact that it is neither prospective nor randomized. A subsequent prospective clinical trial of this technique should be considered because it might lend even stronger support to the desirability of creating a pleural tent at the time of upper lobectomy. Nevertheless, the excellent, consistent results with pleural tents in the current patient population support this clinical strategy as an adjunct to accelerating air leak closure. On the basis of our highly favorable experience, we recommend that surgeons consider the use of routine creation of a pleural tent on patients whenever technically possible at the time they undergo major upper lung resections.

\section{Addendum}

Since the original submission of this manuscript in November, 1996 until July, 1997, 18 more patients (11 men, 7 women; mean age $66.1 \pm 2.4$ years, median 67 years) have undergone an isolated upper lobectomy with creation of a pleural tent at our hospital using the same techniques described herein. There was no mortality or significant morbidity in this subsequent group. The mean total chest tube duration for this additional group was $3.6 \pm 0.4$ days (median 3 days) and the total hospitalization time was $6.3 \pm 0.3$ days (median 6 days). These data compare favorably to the tented group data in Table III, indicating that this technique continues to provide excellent results.

\section{REFERENCES}

1. Daintlth J, Egerton H, Fergusson R, Stibbs A, Wright E, editors. The MacMillan dictionary of quotations. 2nd ed. New York: MacMillan; 1989. p. 5.

2. Barker WI. Natural history of residual air spaces after pulmonary resection. Chest Surg Clin North Am 1996;6:585613.

3. Miller J. Acute and delayed space problems following pulmonary resection. Chest Surg Clin North Am 1996;6:615-21.

4. Fishman NH. Thoracic drainage. Chicago: Year Book; 1983. p. 95-6.

5. Rice TW, Kirby TJ. Prolonged air leak. Chest Surg Clin North Am 1992;2:803-11.

6. Miscall L, Duffy RW, Nolan RB, Klopstock R. The pleural tent as a simultaneous tailoring procedure in combination with pulmonary resection. Am Rev Tuberc 1956;73:831-52.

7. Hansen JL. Parietal pleurolysis (the pleural tent) as a simultaneous space-reducing procedure in combination with pulmonary resection. Acta Chir Scand 1957;112:485-8. 
8. Rainer WG, Newby JP. Prevention of residual space problems after pulmonary resection. Am J Surg 1967;114:744-7.

9. Glantz S. Primer of biostatistics. Version 3.0. New York: McGraw Hill; 1992.

10. Goodman PC, Minagi H, Thomas AN. Radiographic appearance of the chest after pleural space reduction procedures: construction of a pleural tent and phrenoplasty. Am J Roentgenol 1977;129:229-31.

11. Moriarity C. St. Anthony's DRG optimizer. Reston (VA): St. Anthony Publishing; 1997. p. 61.

12. Brewer LA III, Bai AF, Jones WMG. The development of the pleural partition to prevent overexpansion of the lung following partial pulmonary resection. J Thorac Surg 1956; 31:165-82.

13. Rainer WG, Newby JP. Prevention of residual space problems after pulmonary resection. Am J Surg 1968;114:744-7.

14. Brewer LA III, Gazzaniga AB. Phrenoplasty, a new operation for the management of pleural dead space following pulmonary resection. Ann Thorac Surg 1968;6:119-24.

15. Bell JW. Management of the postresection space in tuberculosis. III. Role of pre- and postresection thoracoplasty. J Thorac Surg 1956;31:580-92.

16. Cooper JD, Patterson GA, Sundaresan R, et al. Results of 150 consecutive bilateral lung volume reduction procedures in patients with severe emphysema. J Thorac Cardiovasc Surg 1996;112:1319-30.

\section{Discussion}

Dr. Joseph I. Miller, Jr. (Atlanta, Ga.). Dr. Robinson has refocused our attention on a procedure not often mentioned in the surgical literature since the 1950s and 1960s, as shown in his historical data, except with the reemergence of lung volume reduction surgery when it was used routinely in some centers and selectively in others to prevent apical air leaks.

A number of methods alleviate postresectional spaces; this is one of easiest to achieve as mentioned by Dr. Deslauriers at the postgraduate course. The significance and conclusions of this presentation is a given because there is a reduction in hospital stay and subsequent decrease in total cost to the patient. The two major contributing factors to hospital stay after major thoracotomy and resection are prolonged air leaks and postoperative pain, and the authors are to be congratulated on reducing the hospital stay in their patients by 2 days.

I would raise several questions. I doubt that an apical tent is indicated in all upper lobectomies. In general, I have found that upper lobectomies do not, in general, have an increased frequency of leak compared with lower lobectomies, particularly when the middle lobe is left in place and can conform to the apical space.

I would suggest that perhaps it might be important to reserve this procedure for two potentials. One, after reexpansion of the lung if there is an obvious residual space, then obviously it could be done, or, second, when microleaks are still present despite careful attempts, then perhaps it should be done.

In their manuscript, the authors also state that they routinely cover the lobar stump with a pleural flap. I doubt that this is really necessary. In slightly more than 2000 lobectomies, I have only had one central bronchial leak after this.
A third point is in regard to the patient who has the tented procedure, the residual apical space more often fills with fluid and becomes opacified. At least in my experience, it rarely is obliterated by the remaining lung.

The authors have done an excellent job of referencing the historical evolution of the apical tent and other space-obliterating procedures, and their reference list at the time of publication will be of great interest to residents in the field. I think it is significant that the authors have reintroduced a technique that is known by few thoracic surgical residents today.

I would conclude by asking two questions. One, should the tent be used routinely or selectively? Have the authors also looked at other methods of pain control in the postoperative period to affect decreased hospital stay? Their mean chest tube time was 4 days or did it still require longer?

At present, we have developed a clinical path to discharge patients on day 5 or 6 with a Heimlich valve and a chest tube if they live in the immediate area. Our main problem again has been pain control despite being able to get the chest tube out early.

Dr. Jean Deslauriers (Sainte-Foy, Quebec, Canada). In this article, the authors present comparative data suggesting that routine creation of pleural tent done at the time of upper lobectomy significantly reduces the time that chest tubes remain in place postoperatively and, therefore, results in shorter hospital stays.

Although I agree that pleural tenting is a simple procedure that could be a valid addition to upper lobectomy when a residual space or prolonged air leaks are anticipated, I am not certain that it should be recommended routinely for all cases.

The reasons why I question this recommendation are threefold and basically are the same issues raised by Dr. Clagett when he discussed the use of parietal pleurectomy to treat spontaneous pneumothoraces.

The first may be occasional early complications related to the tent such as hemorrhage or infection developing within the extrapleural space.

Second, the lung may not reexpand well, leading to significant pleural thickening and possibly some restriction to normal breathing.

Third, if reoperation ever becomes necessary, the previously created tent may make redo surgery very hazardous.

I would like to ask Dr. Robinson if he has looked at all 3- and 6-month radiographs in this cohort of patients to see how many have significant apical thickening and if he has ever had to reoperate on one of these patients.

Dr. Paul A. Kirschner (New York, N.Y.). I would like to add a historical note to this, having been a resident under Dr. Lawrence Miscall. He was one of our consultants at the Bronx VA when the VA residency program first started in the late 1940s after World War II.

The main reason for the pleural tent was not prolonged air leak or prolonged drainage, it was to prevent overexpansion of the lung after resection for tuberculosis. The theory at that time was that tuberculous foci in the lung would break down if the lung overexpanded.

So there were two ways of controlling this situation after lung resection, lobectomy, either so-called tailoring 
thoracoplasty or, as Dr. Miscall introduced, the "pleural tent. "1 And we did it the same way that was described by the author today, but not for the same reason. We did not have any problems with infection in the space, tuberculous or otherwise, and the usual experience was that this space filled with fluid, which was gradually absorbed so that ultimately, with the passage of time, you would just see a thickening, a little apical cap, at the site and not very much trace of the tent as the years went by.

\section{REFERENCE}

1. Miscall L, Duffy RW, Nolan RB, Klopstock R. The pleural tent as a simultaneous tailoring procedure in combination with pulmonary resection. Am Rev Tuberc 1956;73:831-52.

Dr. Robinson. I would like to thank the discussants for their comments. Dr. Miller, I appreciate your comments and I have read and appreciated your recent paper on postoperative pleural space problems. This review was published recently and has a fine discussion of the management of postoperative pleural spaces.

I thought that when I made the comment regarding routine use of the pleural tent I would probably raise some eyebrows. I have become quite used to doing this procedure and it takes approximately 3 or 4 minutes. I have not had problems with it. There is essentially no associated bleeding from the pleura. You look around intraoperatively to try to find any bleeding points on the parietal pleura, but that has not been a problem. We also have not had problems with pleural infections.

One of the difficulties when you reexpand the lung after a resection is that usually it is hard to tell looking at the lung how much residual air space will be left. Consequently, it is often difficult to tell whether you should use a pleural tent based on this criterion. I have become very accustomed to doing a pleural tent and relying on it to close the air leaks and to have my chest tubes out quickly postoperatively. Therefore I have added this procedure routinely to my upper lobectomies. I also do pleural tents on large segmentectomies, particularly in emphysematous lungs.

I think the pleural tent should be considered as another method to have available in the surgeon's armamentarium, which may be used from time to time. I suspect most surgeons would use this technique selectively.

The pain control method that we use is a continuous epidural placed by our anesthesiologists. We find that this works very well and have not had much problem with pain control. Occasionally we use some intravenous ketorolac tromethamine as a lot of other people do also. We are also able to discharge patients fairly quickly. This series was collected over a 21/2-year period. We send patients home a lot quicker nowadays, usually at around 4 or 5 days postoperatively. Most of our patients' chest tubes are out on one day and they are home the next day.

I might mention that I have some reservations about using a Heimlich valve to discharge patients with a chest tube. I think this device no longer gives you a closed system. I have had concerns that if you do have a pleural space, you may end up introducing retrograde infection through the Heimlich valve. So I have not used the Heimlich valve. Whether infection of the space will occur, I am not sure, but I have not felt comfortable with this device.

Dr. Deslauriers mentioned the possibility of having an occasional complication from the pleural tent itself. We have not had any problems so far.

And I appreciate Dr. Kirschner's remarks regarding his experience with Dr. Miscall. This technique described by Dr. Miscall appears to work very well, and the concerns about hemorrhage and empyema have not been a problem in our experience. I think that was the experience back in the 1950s and 1960s. I have not had an opportunity to operate on someone again who has had a previous pleural tent, but I will be interested to see what it is like. It is hard to believe it would be any worse than the usual redo thoracotomy. Therefore, as far as reoperation is concerned, I do not anticipate this being a problem.

I should also mention that the pleural flap that I put over the bronchial stump is something that I was taught to do as a resident. I have not ever had a problem with a bronchopleural fistula when I used a pleural flap, and I am afraid to stop using it now. So I will continue despite the fact that it may not make a difference.

Again, I appreciate Dr. Kirschner's interesting historical remarks about Dr. Miscall. He is correct that it was lung overexpansion that used to be the concern, and this is why it was thought that you needed to collapse the chest wall with a thoracoplasty in the 1940s and 1950s. You wanted to try to prevent overexpansion of the lung. Later, obviously, it was realized that this was not a problem and collapse was not necessary. 